\title{
Damage theory: microscopic effects of vanishing macroscopic motions
}

\author{
ELENA BONETTI ${ }^{1}$ and MICHEL FRÉMOND ${ }^{2}$ \\ ${ }^{1}$ Laboratoire Lagrange, Dipartimento di Matematica "F. Casorati", \\ Università di Pavia, Via Ferrata 1, 27100 Pavia, Italy \\ E-mail: bonetti@dimat.unipv.it \\ ${ }^{2}$ Laboratoire Lagrange, Laboratoire Central des Ponts et Chaussées \\ 58 boulevard Lefebvre, 75732 Paris Cedex 15, France \\ E-mail: michel.fremond@1cpc.fr
}

\begin{abstract}
This paper deals with a mechanical model describing the evolution of damage in elastic and viscoelastic materials. The state variables are macroscopic deformations and a microscopic phase parameter, which is related to the quantity of damaged material. The equilibrium equations are recovered by refining the principle of virtual powers including also microscopic forces. After proving an existence and uniqueness result for a regularized problem, we investigate the behavior of solutions, in the case when a vanishing sequence of external forces is applied. By use of a rigorous asymptotics analysis, we show that macroscopic deformations can disappear at the limit, but their damaging effect remains in the equation describing the evolution of damage at a microscopic level. Moreover, it is proved that the balance of the energy is satisfied at the limit.
\end{abstract}

Mathematical subject classification: 74A45, 73C50, 35K60.

Key words: damage, principle of virtual power, non linear elasticity, nonlinear parabolic PDE, asymptotic analysis.

\section{Introduction}

These notes are concerning with a mechanical model describing the evolution of damage in elastic and viscoelastic bodies (cf. [4, 8]). It is known that the mechanical process of damage results from microscopic motions in the structure, as it is caused by microfractures and microcavities resulting in the decreasing of 
the material stiffness. Consequently, to provide a good macroscopic predictive theory one has to account for macroscopic effects of microscopic motions. In particular, the model is written in terms of a damage quantity within the framework of continuum mechanics and we derive the constitutive equations by two functionals, the free energy and the pseudo-potential of dissipation (cf. [11]), which are responsible for the equilibrium and the evolution of the system, respectively. The constitutive equations are coupled with equations of motion resulting from the principle of virtual power. The main idea in this modeling procedure consists in refining the power of interior forces by including also the effects of microscopic movements, which are clearly related to the damage rate. We recall that, when compared to experimental results, the model can predict the behavior of concrete structure correctly and, accounting for the gradient of damage, the structure size effect, too, which is particularly important in civil engineering (cf. [12]).

Concerning the analytical investigation of the above model, as far as we know, only some partial results have been obtained. The difficulty is mainly due to the degeneracy of the elastic coefficient in the macroscopic equilibrium equation. This is combined in the equations of microscopic motions, with the presence of subdifferential operators and quadratic nonlinearity for deformations. In $[6,7]$ a simplified version of the problem is investigated in the one-dimensional case, regularizing the subdifferentials. In particular, local in time existence and uniqueness results are proved both for the quasi-static situation and in the case when macroscopic accelerations are retained in the principle of virtual powers. Finally, we recall a recent paper [3] dealing with the complete 3D problem, but describing the evolution of damage only during the time interval in which the material is not completely damaged. A local in time existence result is stated.

However, these notes do not focus on the problem of existence of solutions, but mainly investigate relations between macroscopic and microscopic motions during the damaging process. Indeed, as the modeling method is based on the separation between the description of macroscopic and microscopic levels, it is natural to wonder which are the microscopic effects of vanishing macroscopic motions. Concerning this subject, in a recent paper [5], it is presented an example of vanishing macroscopic motions which retain at the limit a damaging effect 
in the microscopic equation. Now, we aim to show that the above behavior can be proved in a more general situation. The main idea consists in approximating the system to get a global existence and uniqueness result, and then applying a vanishing sequence of exterior forces in such a way that the resulting macroscopic motions become smaller and smaller. By use of a rigorous asymptotics analysis we show that at the limit the macroscopic motions become microscopic, in the sense that the macroscopic motions vanish but their effects remain as a source of damage, which is clearly related to microscopic motions. Moreover, we show that the balance of the energy is satisfied at the limit.

\section{The model}

In this section, we briefly recall the model and write the corresponding PDE's system. Concerning the mechanical aspects, we do not enter the details and refer, e.g. to [4]. Nonetheless, for the sake of completeness, let us point out the main ingredients of the modeling procedure. Assuming that the temperature is constant, the state variables are the strain tensor $\varepsilon(\mathbf{u})$ (u is the vector of small displacements), a phase parameter $\chi$ denoting the quantity of damaged material, and the gradient $\nabla \chi$ accounting for local microscopic interactions. Concerning the phase parameter $\chi$, we prescribe the constraint

$$
\chi \in[0,1],
$$

letting $\chi=1$ correspond to the undamaged structure and $\chi=0$ to the completely damaged material. Next, the dissipation due to damage is reasonably described by the dissipative variables $\chi_{t}, \nabla \chi_{t}$ related to microscopic velocities, where the subscript ${ }_{t}$ denotes the time derivative. After introducing the free energy functional and a pseudo-potential of dissipation, e.g. as in $[4,8]$, we can explicitly write in terms of the unknowns $(\mathbf{u}, \chi)$ the constitutive laws for microscopic and macroscopic stresses. Finally, we state the balance law for momentum, both for microscopic and macroscopic motions. More precisely, we consider a concrete structure located in a bounded smooth domain $\Omega \subset \mathbf{R}^{3}$ with boundary $\partial \Omega=: \Gamma$. We look at the damage evolution during a finite time interval $[0, T]$ and denote by $Q:=\Omega \times[0, T]$. Hence, the principle of virtual power, in which we include also microscopic forces, leads to the following inclusion governing the evolution 
of the phase parameter

$$
c \chi_{t}-k \Delta \chi+\partial I_{[0,1]}(\chi) \ni w-\frac{1}{2}|\varepsilon(\mathbf{u})|^{2},
$$

whose ingredients will be specified in a moment, and to the equilibrium equation for macroscopic deformations, written in the quasi-static case

$$
\operatorname{div}(\chi \varepsilon(\mathbf{u}))+\mathbf{f}=\mathbf{0} .
$$

The positive constants $c, k$, and $w$ in (2.2) denote the viscosity of damage, a local interaction coefficient, and the cohesion energy, respectively. The term $\partial I_{[0,1]}$ is the subdifferential of the indicator function $I_{[0,1]}$ and it accounts for the required constraint on $\chi$ prescribed by (2.1). Indeed, the domain $\partial I_{[0,1]}$ is the interval $[0,1]$, and we have $\partial I_{[0,1]}(y)=0$ if $y \in(0,1), \partial I_{[0,1]}(0)=(-\infty, 0]$, and $\partial I_{[0,1]}(1)=[0,+\infty)$. In $(2.3), \mathbf{f}$ denotes a volumic force applied to the structure. Nonetheless, in the following we consider a regularized version of the above equation, which is obtained by adding a viscosity contribution for macroscopic displacements and deformations, and an higher order nonlinear elastic term in the stress. More precisely, we substitute (2.3) by

$$
\mathbf{u}_{t}-\operatorname{div}\left(\varepsilon\left(\mathbf{u}_{t}\right)\right)-\operatorname{div}\left(|\varepsilon(\mathbf{u})|^{2} \varepsilon(\mathbf{u})+\chi \varepsilon(\mathbf{u})\right)=\mathbf{f} .
$$

Remark 2.1. Let us not that from a mechanical point of view (2.4) is associated with a viscous phenomenon, by the presence of the velocity terms $\mathbf{u}_{t}$ and $\varepsilon\left(\mathbf{u}_{t}\right)$.

Remark 2.2. Let us stress that equations (2.2) and (2.3) or (2.4) are coupled: the macroscopic motion, i.e., displacement $\mathbf{u}$, influences the microscopic motion schematised by $\chi$ and governed by equation (2.2). Conversely, microscopic motion when resulting from a damage source, i.e., a right hand side in (2.2) influences macroscopic motion $\mathbf{u}$ governed by equation (2.3) or (2.4).

Remark 2.3. We point out that the regularizing (2.3) by (2.4) turns out to be useful to control the right hand side of (2.2). Indeed, as the elasticity coefficient $\chi$ in (2.3) degenerates once the material is completely damaged, i.e. $\chi=0$, the right hand side of (2.2) could not be controlled. In terms of mechanics, the 
regularization (2.4) controls the strain localisation which may occur in damage phenomena and preserves the uniqueness of solutions.

Now, we have to prescribe suitable initial and boundary conditions. Assuming, for the sake of simplicity, that at the initial time $t=0$ the material is completely undamaged, we prescribe

$$
\chi(0)=1 \quad \text { a.e. in } \Omega \text {. }
$$

Next, we assume that no energy is provided from the outside (cf. [4])

$$
\partial_{n} \chi=0 \quad \text { a.e. in } \Gamma \times(0, T) .
$$

Finally, we fix an initial condition on the vector of displacements $\mathbf{u}$

$$
\mathbf{u}(0)=\mathbf{0},
$$

and, letting $\mathbf{n}$ be the outward normal unit vector to the boundary, we give a Neumann condition on the stress

$$
\left(\varepsilon\left(\mathbf{u}_{t}\right)+|\varepsilon(\mathbf{u})|^{2} \varepsilon(\mathbf{u})+\chi \varepsilon(\mathbf{u})\right) \mathbf{n}=\mathbf{0} \quad \text { on } \Gamma .
$$

from which it follows that the applied traction on the boundary is $\mathbf{0}$.

In the following, we are interested to consider the behavior of the solutions $(\chi, \mathbf{u})$ to an initial and boundary values problem associated with (2.2), (2.4), in the case when a vanishing sequence of external forces $\mathbf{f}_{\tau}$ is applied, i.e. $\mathbf{f}_{\tau} \searrow \mathbf{0}$ as $\tau$ tends to 0 . Hence, letting $\left(\mathbf{u}_{\tau}, \chi_{\tau}\right)$ be the corresponding solutions, we can exploit an a priori estimates - passage to the limit procedure to perform the required asymptotics analysis as $\tau \searrow 0$. Thus, at a first step, we aim to find existence and uniqueness of solutions to the system for any fixed $\tau>0$ and, on a second step, prove boundedness properties on these solutions which allow us to pass to the limit as $\tau \searrow 0$.

Here is the outline of the paper. In Section 3, we introduce the abstract formulation of the regularized system and state the main existence and uniqueness theorem (cf. Theorem 3.1). Hence, in Section 4, under suitable assumptions on the convergence of a sequence of applied forces $f_{\tau}$, we perform an asymptotics analysis of the solutions as $\tau \searrow 0$ (cf. Theorem 4.1). Finally, in Section 5, we investigate the balance of the energy at the limit $\tau=0$. 


\section{The abstract problem}

In this section, we first introduce the abstract version of the regularized damage system (2.2), (2.4) combined with initial and boundary conditions (2.5)-(2.6), (2.7)-(2.8). We prove that the resulting problem admits a unique solution and then, in the next sections, we investigate the asymptotics behavior of the solution as the external efforts applied to the structure vanish. Let us first introduce a suitable abstract setting. In regards of simplicity, but without loss of generality, in the following we take $\mathbf{u}$ as a scalar quantity $u$. In addition, fix $c=k=1$ in (2.2). Hence, we start by considering the Hilbert triplet (cf. [10])

$$
V \hookrightarrow H \hookrightarrow V^{\prime}
$$

where

$$
V:=H^{1}(\Omega) \quad \text { and } \quad H:=L^{2}(\Omega)
$$

$H$ being identified as usual with its dual space. Notice that we use the same notation $\|\cdot\|_{X}$ both for the norm in a functional space $X$ and in $X^{3}$. Hence, let

$$
W:=W^{1,4}(\Omega) .
$$

Finally, we introduce the following abstract operators

$$
\begin{aligned}
& A: V \rightarrow V^{\prime} \quad{ }_{V^{\prime}}\langle A u, v\rangle_{V}=\sum_{i=1}^{3} \int_{\Omega} D_{i} u D_{i} v=\int_{\Omega} \nabla u \cdot \nabla v \quad u, v \in V, \\
& \mathcal{A}: W \rightarrow W^{\prime} \quad{ }_{W^{\prime}}\langle\mathcal{A} u, v\rangle_{W}=\sum_{i=1}^{3} \int_{\Omega}\left|D_{i} u\right|^{2} D_{i} u D_{i} v \quad u, v \in W, \\
& B:\left(L^{4 / 3}(\Omega)\right)^{3} \rightarrow W^{\prime} \quad{ }_{W^{\prime}}\langle B \mathbf{u}, v\rangle_{W}=\sum_{i=1}^{3} \int_{\Omega} u_{i} D_{i} v=\int_{\Omega} \mathbf{u} \cdot \nabla v, \\
& \mathbf{u} \in\left(L^{4 / 3}(\Omega)\right)^{3}, v \in W,
\end{aligned}
$$

where $D_{i}$ stands for the usual partial derivative operator with respect to the variable $x_{i}, i=1,2$, 3. In particular, let us observe that (3.1) corresponds to the laplacian operator with associated Neumann homogeneous boundary condition 
(cf. (2.6)) in the duality pairing between $V^{\prime}$ and $V$. Analogously, (3.2) corresponds to the abstract formulation of the 4-laplacian operator with associated homogeneous boundary assumption. Now, we can make precise the abstract problem we are dealing with and state a corresponding existence and uniqueness result.

Problem $P_{a}:$ Find $(u, \chi)$ such that

$$
u(0)=0, \quad \chi(0)=1 \quad \text { a.e. in } \Omega,
$$

and fulfilling for a.a. $t$ in $(0, T)$

$$
\begin{aligned}
& u_{t}+A u_{t}+\mathcal{A} u+B(\chi \nabla u)=f \quad \text { in } W^{\prime}, \\
& \chi_{t}+A \chi+\xi=w-\frac{1}{2}|\nabla u|^{2} \quad \text { in } H,
\end{aligned}
$$

where $\xi \in L^{2}(0, T ; H)$ is such that $\xi \in \partial I_{[0,1]}(\chi)$ a.e. in $Q$.

The following proposition holds.

Theorem 3.1. Let

$$
f \in L^{2}\left(0, T ; V^{\prime}\right) \cap L^{1}(0, T ; H) .
$$

Then, Problem $P_{a}$ admits a unique solution $(u, \chi)$ with regularity

$$
\begin{aligned}
& u \in H^{1}(0, T ; V) \cap L^{\infty}\left(0, T ; H^{2}(\Omega)\right), \\
& \chi \in H^{1}(0, T ; H) \cap L^{\infty}(0, T ; V) \cap L^{2}\left(0, T ; H^{2}(\Omega)\right) .
\end{aligned}
$$

Proof. To prove the existence of a solution to Problem $P_{a}$ stated by Theorem 3.1, we mainly exploit a fixed point argument and refer to well known results on evolution nonlinear equations. In regard of simplicity, we do not enter the details of the demonstration, but just sketch the main idea and give some useful references. We aim to apply the Schauder theorem to a suitable operator we are going to construct as it follows. We start by letting

$$
\widehat{u} \in X, \quad X:=\left\{u \in L^{4}(0, T ; W) \text { with }\|u\|_{L^{4}(0, T ; W)} \leq R\right\},
$$


where $R$ will be fixed later, and substitute $\nabla u$ in (3.6) by $\nabla \widehat{u}$. Thus, after observing that by the Hölder inequality the right hand side of (3.6) is in $L^{2}(Q)$, we can apply well-known results on evolution equations associated with maximal monotone operators (cf. e.g. [2]). We infer that there exists a unique corresponding solution

$$
\begin{aligned}
& \chi:=E(\widehat{u}) \in H^{1}(0, T ; H) \cap L^{\infty}(0, T ; V) \cap L^{2}\left(0, T ; H^{2}(\Omega)\right), \\
& \chi \in[0,1] \text { a.e. in } Q .
\end{aligned}
$$

Moreover, one can prove that the following estimate

$$
\|\chi\|_{H^{1}(0, T ; H) \cap L^{\infty}(0, T ; V) \cap L^{2}\left(0, T ; H^{2}(\Omega)\right)} \leq c,
$$

holds for a suitable constant $c$ depending on $\Omega, T, w$, and $R$, but not on the choice of $\widehat{u}$ in (3.10). Let us briefly outline the procedure and prove some formal a priori estimates on the solution. We test (3.6) by $\chi_{t}$ and integrate over $(0, t)$. After an application of the Young inequality

$$
a b \leq \frac{1}{2 \delta} a^{2}+\frac{\delta}{2} b^{2}, \quad a, b \in \mathbf{R}, \delta>0,
$$

and integrating by parts in time, we have (cf. (3.4))

$$
\begin{aligned}
\left\|\chi_{t}\right\|_{L^{2}(0, t ; H)}^{2} & +\|\nabla \chi(t)\|_{H}^{2}+\int_{\Omega} I_{[0,1]}(\chi(t)) \\
& \leq c\left(1+\int_{0}^{t} \int_{\Omega}|\nabla \widehat{u}|^{4}\right) \leq c .
\end{aligned}
$$

In particular, let us point out that in deducing (3.14), one can formally proceed as follows

$$
\begin{aligned}
& \int_{0}^{t} \int_{\Omega} \xi \chi_{t}=\int_{0}^{t} \int_{\Omega} \frac{d}{d t} I_{[0,1]}(\chi)=\int_{\Omega} I_{[0,1]}(t), \\
& \int_{0}^{t} \int_{\Omega}|\nabla \widehat{u}|^{2} \chi_{t} \leq \frac{1}{2} \int_{0}^{t} \int_{\Omega}\left|\chi_{t}\right|^{2}+\frac{1}{2} \int_{0}^{t} \int_{\Omega}|\nabla \widehat{u}|^{4} .
\end{aligned}
$$

Then, we test (3.6) by $A \chi$ and recall that, by monotonicity of the subdifferential operator and the chain rule, it follows

$$
\int_{0}^{t} \int_{\Omega} \xi A \chi \geq 0
$$


Thus, by similarly proceeding as in (3.14) we get

$$
\|A \chi\|_{L^{2}(0, t ; H)} \leq c .
$$

Moreover, owing to (3.10), (3.14), and (3.18), a comparison in (3.6) leads to

$$
\|\xi\|_{L^{2}(0, T ; H)} \leq c
$$

On a second step, we fix $\chi=E(\widehat{u})$ in (3.5) and look for a corresponding solution fulfilling the required Cauchy condition stated by (3.4). Thanks to (3.7) and (3.11), we owe, e.g., to [9, Theorem 1.2bis, p. 163-164] to infer existence and uniqueness of a unique solution

$$
u:=G(\chi)
$$

Hence, let us show that for a suitable choice of $R$ in (3.10), $u=G(E(\widehat{u}))$ turns out to belong to $X$. Indeed, we can test (3.5) by $u$ and integrate in time over $(0, t)$. Recalling (3.1)-(3.4), a suitable application of the Young inequality yields

$$
\begin{aligned}
\|u(t)\|_{V}^{2} & +\int_{0}^{t} \sum_{i=1}^{3} \int_{\Omega}\left|D_{i} u\right|^{4}+\int_{0}^{t} \int_{\Omega} \chi|\nabla u|^{2} \\
& \leq c \int_{0}^{t}\|f\|_{V^{\prime}}\|u\|_{V} \leq c\left(\|f\|_{L^{2}\left(0, t ; V^{\prime}\right)}^{2}+\|u\|_{L^{2}(0, t ; V)}^{2}\right),
\end{aligned}
$$

and the Gronwall lemma yields

$$
\|u\|_{L^{\infty}(0, T ; V)}+\|u\|_{L^{4}(0, T ; W)} \leq \widetilde{c},
$$

where $\widetilde{c}$ depends only $\Omega, T$, and $f$. Thus, if we choose $R \geq \widetilde{c}$ in (3.10), it follows that

$$
P: X \rightarrow X, \quad P(\widehat{u}):=G(E(\widehat{u}))
$$

maps $X$ into itself. Then, towards the aim of applying the Schauder theorem to the operator $X$, and consequently prove that $P$ admits a fixed point, we show that $P$ is a continuous and compact operator in $X$ with respect to the natural topology induced by $L^{4}(0, T ; W)$. We perform some a priori estimates, which turn out to be useful to prove both the compactness and the continuity of $P$. Let us point 
out that some of the following estimates are only formal. Nonetheless, they can be rigorously justified prooceeding, e.g., as in [9].

We first test (3.5) by $u_{t}$ and then integrate over $(0, t)$. Hence, integrating by parts in time and applying the Young inequality, we infer that

$$
\begin{aligned}
\left\|u_{t}\right\|_{L^{2}(0, t ; V)}^{2} & +\frac{1}{4} \sum_{i=1}^{3}\left\|D_{i} u(t)\right\|_{L^{4}(\Omega)}^{4}+\frac{1}{2} \int_{\Omega} \chi(t)|\nabla u(t)|^{2} \\
& \leq c\left(\int_{0}^{t} \int_{\Omega} f u_{t}+\int_{0}^{t} \int_{\Omega} \chi_{t}|\nabla u|^{2}\right) \leq \frac{1}{2}\left\|u_{t}\right\|_{L^{2}(0, t ; V)}^{2} \\
& +c\left(\|f\|_{L^{2}\left(0, T ; V^{\prime}\right)}^{2}+\left\|\chi_{t}\right\|_{L^{2}(0, t ; H)}^{2}+\sum_{i=1}^{3} \int_{0}^{t}\left\|D_{i} u\right\|_{L^{4}(\Omega)}^{4}\right) .
\end{aligned}
$$

In particular, let us point out that we have performed the following integration by parts in time

$$
\begin{aligned}
\int_{0}^{t} \int_{\Omega} \chi_{t} \nabla u \cdot \nabla u_{t} & =\frac{1}{2} \int_{0}^{t} \int_{\Omega} \chi \frac{d}{d t}|\nabla u|^{2} \\
& =\frac{1}{2} \int_{\Omega} \chi(t)|\nabla u(t)|^{2}-\frac{1}{2} \int_{0}^{t} \int_{\Omega} \chi_{t}|\nabla u|^{2} .
\end{aligned}
$$

Thus, after recalling that (3.12) holds independently of $\widehat{u}$, the Gronwall lemma yields

$$
\left\|u_{t}\right\|_{L^{2}(0, T ; V)}+\sum_{i=1}^{3}\left\|D_{i} u\right\|_{L^{\infty}\left(0, T ; L^{4}(\Omega)\right)} \leq c .
$$

Finally, we formally test (3.5) by $A u$. By monotonicity arguments and applying the chain rule, we have

$$
{ }_{W^{\prime}}\langle\mathcal{A} u, A u\rangle_{W} \geq 0
$$

Then, by definition of the operators $A$ and $B$, owing to (3.12), (3.7), and (3.27), we can write

$$
\begin{aligned}
& \frac{1}{2}\|\nabla u(t)\|_{H}^{2}+\frac{1}{2}\|A u(t)\|_{H}^{2}+\int_{0}^{t} \int_{\Omega} \chi|A u|^{2} \\
& \quad \leq c\left(\int_{0}^{t}\|\nabla \chi\|_{L^{4}(\Omega)}\|\nabla u\|_{L^{4}(\Omega)}\|A u\|_{H}+\int_{0}^{t}\|f\|_{H}\|A u\|_{H}\right) .
\end{aligned}
$$


Thus, owing to the regularity of $\chi$ (cf. in particular (3.18)) and (3.26), the generalized version of the Gronwall introduced in [1] ensures

$$
\|A u\|_{L^{\infty}(0, T ; H)} \leq c,
$$

and finally

$$
\|u\|_{L^{\infty}\left(0, T ; H^{2}(\Omega)\right)} \leq c .
$$

Thus, we can infer that

$$
\|P(\widehat{u})\|_{H^{1}(0, T ; V) \cap L^{\infty}\left(0, T ; H^{2}(\Omega)\right)} \leq c,
$$

from which it easily follows that $P$ is a compact operator in $X$. Moreover, the previous estimates (3.12) and (3.31), which are independent of the choice of $\widehat{u}$, allow us to deduce that $P$ is a continuous operator in $X$. Towards the aim of proving continuity of $P$, we take

$$
\widehat{u}_{n} \in X, \quad \widehat{u}_{n} \rightarrow \widehat{u} \quad \text { in } L^{4}(0, T ; W),
$$

and set

$$
\chi_{n}=E\left(\widehat{u}_{n}\right), \quad u_{n}=G\left(\chi_{n}\right) .
$$

Our purpose is to show that

$$
P\left(\widehat{u}_{n}\right) \rightarrow P(\widehat{u}),
$$

strongly in $L^{4}(0, T ; W)$ (cf. (3.32)). Proceeding as above, we show that $\left(u_{n}, \chi_{n}, \xi_{n}\right)$, with $\xi_{n} \in \partial I_{[0,1]}\left(\chi_{n}\right)$, fulfil the following estimate (cf. (3.12) and (3.31))

$$
\begin{aligned}
& \left\|\chi_{n}\right\|_{H^{1}(0, T ; H) \cap L^{\infty}(0, T ; V) \cap L^{2}\left(0, T ; H^{2}(\Omega)\right)}+\left\|\xi_{n}\right\|_{L^{2}(0, T ; H)} \\
& \quad+\left\|u_{n}\right\|_{H^{1}(0, T ; V) \cap L^{\infty}\left(0, T ; H^{2}(\Omega)\right)} \leq c,
\end{aligned}
$$

independently of $n$. By weak and weak star compactness results, at least for some suitable subsequence, the following convergence holds

$$
\chi_{n} \stackrel{*}{\rightarrow} \chi \quad \text { in } H^{1}(0, T ; H) \cap L^{\infty}(0, T ; V) \cap L^{2}\left(0, T ; H^{2}(\Omega)\right),
$$


and by compactness (cf. [13])

$$
\chi_{n} \rightarrow \chi \quad \text { in } C^{0}([0, T] ; H) \cap L^{2}(0, T ; V) .
$$

Hence, by the boundedness of $\xi_{n}$ stated by (3.35), we have

$$
\xi_{n} \rightarrow \xi \quad \text { in } L^{2}(0, T ; H),
$$

so that (3.37) and (3.38) ensure $\xi \in \partial I_{[0,1]}(\chi)$ (cf. [2]). Now, we can pass to the limit in (3.6), written for $n$, as $n \rightarrow+\infty$, and by the uniqueness of the solution for the limit equation, once $\widehat{u}$ is fixed, we eventually identify $\chi=E(\widehat{u})$. Moreover, let us observe that uniqueness of the solution for the limit equation extends convergences (3.36)-(3.38) to the whole sequences. On a second step, we discuss the asymptotics behavior of $u_{n}$ and $\left|\nabla u_{n}\right|^{2}$, in order to pass to the limit in (3.5) written for $n$. By (3.35), we deduce, at least up to the extraction of some subsequence,

$$
\begin{aligned}
& u_{n} \stackrel{*}{\rightarrow} u \quad \text { in } H^{1}(0, T ; V) \cap L^{\infty}\left(0, T ; H^{2}(\Omega)\right), \\
& \left|\nabla u_{n}\right|^{2} \rightarrow d \quad \text { in } L^{\infty}(0, T ; H) .
\end{aligned}
$$

By compactness, (3.39) leads to the strong convergence

$$
u_{n} \rightarrow u \quad \text { in } L^{\infty}(0, T ; W),
$$

which allows us to eventually identify $d=|\nabla u|^{2}$. Hence, to show that $P$ is continuous, we aim to pass to the limit as $n \rightarrow+\infty$ in (3.5), which is possible thanks to (3.39), (3.40), and (3.41). Then, uniqueness of the solution for the limit equation allow us to identify $u=G(\chi)$ in (3.41), concludes the proof. Moreover, it follows that (3.39)-(3.41) actually hold for the whole sequence. In particular, $P$ turns out to satisfy the assumptions of the Schauder theorem and consequently it admits a fixed point $u$. It is now a standard matter to verify that the couple of functions $(u, \chi=E(u))$ is a solution to Problem $P_{a}$.

Hence, to complete the proof of Theorem 3.1, it remains to show that this solution is unique. First let us make some few remarks about useful notation we will use in the sequel. We assume that Problem $P_{a}$ admits two solutions

$$
S_{1}=\left\{u_{1}, \chi_{1}\right\}, \quad S_{2}=\left\{u_{2}, \chi_{2}\right\},
$$


whose regularity is specified by Theorem 3.1 . We denote by $[f]$ the difference of two functions $f_{1}, f_{2}$, i.e.

$$
[f]=f_{1}-f_{2},
$$

and by $\underline{f}$, the mean value

$$
\underline{f}=\frac{f_{1}+f_{2}}{2},
$$

so that we can make use of the identity

$$
f_{1} g_{1}-f_{2} g_{2}=[f g]=[f] \underline{g}+\underline{f}[g] .
$$

To simplify notation, but without loss of generality, in the sequel let us rewrite (3.45) and subsequent computations omitting the symbol of the mean value, namely

$$
[f g]=[f] g+f[g] .
$$

Now, we take the difference of (3.5) written for $S_{1}$ and $S_{2}$

$$
[u]_{t}+A[u]_{t}+[\mathcal{A} u]+B([\chi] \nabla u)+B(\chi \nabla[u])=0,
$$

test by $[u]$, and integrate over $(0, t)$. By monotonicity of $\mathcal{A}$ (cf. [9]), we have

$$
\int_{0}^{t}{ }_{W^{\prime}}\langle[\mathcal{A} u],[u]\rangle_{W} \geq 0 .
$$

Thus, recalling the definition of the operator $B$, and integrating by parts in time, we write

$$
\begin{aligned}
\frac{1}{2}\|[u](t)\|_{V}^{2} & +\int_{0}^{t} \int_{\Omega} \chi|\nabla[u]|^{2} \\
& \leq \int_{0}^{t}\|\nabla u\|_{L^{4}(\Omega)}\|[\chi]\|_{L^{4}(\Omega)}\|\nabla[u]\|_{H} .
\end{aligned}
$$

Analogously, we take the difference of (3.6) written for $S_{1}$ and $S_{2}$, test by [ $\chi$ ], and integrate over $(0, t)$. By monotonicity of the subdifferential operator, there holds

$$
\int_{0}^{t} \int_{\Omega}[\xi][\chi] \geq 0
$$


Thus, we finally write

$$
\frac{1}{2}\|[\chi](t)\|_{H}^{2}+\|\nabla[\chi]\|_{L^{2}(0, t ; H)}^{2} \leq 2 \int_{0}^{t}\|\nabla u\|_{L^{4}(\Omega)}\|\nabla[u]\|_{H}\|[\chi]\|_{L^{4}(\Omega)} .
$$

Now, we can add (3.49) and (3.51), apply the Young inequality to get (cf. (3.8))

$$
\begin{aligned}
& \frac{1}{2}\|[u](t)\|_{V}^{2}+\frac{1}{2}\|[\chi](t)\|_{H}^{2}+\|\nabla[\chi]\|_{L^{2}(0, t ; H)}^{2} \leq \delta\|\nabla[\chi]\|_{L^{2}(0, t ; H)}^{2} \\
& \quad+C_{\delta}\left(\|[\chi]\|_{L^{2}(0, t ; H)}^{2}+\|\nabla u\|_{L^{\infty}\left(0, T ; L^{4}(\Omega)\right)}^{2}\|[u]\|_{L^{2}(0, t ; V)}^{2}\right) \\
& \quad \leq \delta\|\nabla[\chi]\|_{L^{2}(0, t ; H)}^{2}+c\left(\|[\chi]\|_{L^{2}(0, t ; H)}^{2}+\|[u]\|_{L^{2}(0, t ; V)}^{2}\right) .
\end{aligned}
$$

Thus, owing to the Gronwall lemma and taking, e.g., $\delta=1 / 2$ we finally have

$$
[u]=[\chi]=0 \quad \text { a.e. in } Q,
$$

which concludes the proof of uniqueness.

Remark 3.2. It is clear that Theorem 3.1. holds also in the case when the higher order nonlinear elastic term is neglected in (3.5), namely the equation governing macroscopic displacements is substituted by

$$
u_{t}+A u_{t}+B(\chi \nabla u)=f .
$$

Nonetheless, it turns out to be useful to keep it in order to perform the following asymptotics analysis.

Remark 3.3. Let us point out that the presence of the viscosity term involving deformations is strongly exploited in the proof of the uniqueness of the solution to Problem $P_{a}$. Analogously, we could introduce dissipation for the gradient of the phase parameter by adding $A \chi_{t}$ in (3.6).

Remark 3.4. An analogous result as in Theorem 3.1 could be obtained neglecting $u_{t}$ in (3.5), but to substitute the boundary condition (2.8), e.g., by a Dirichlet prescription on the velocity $u_{t}$ on $\Gamma$, to get $A$ be coercive via Poincaré's inequality. 


\section{Passage to the limit for vanishing external forces}

Theorem 3.1 ensures that, for $f$ fulfilling (3.7) there exists a unique solution to Problem $P_{a}$. In this section, we consider a sequence $f_{\tau}$ of exterior forces fulfilling (3.7) and denote by $\left(u_{\tau}, \chi_{\tau}\right)$ the corresponding solution to Problem $P_{a}$, i.e.

$$
\begin{aligned}
& u_{\tau_{t}}+A u_{\tau_{t}}+\mathcal{A} u_{\tau}+B\left(\chi_{\tau} \nabla u_{\tau}\right)=f_{\tau} \\
& \chi_{\tau_{t}}+A \chi_{\tau}+\xi_{\tau}=w-\frac{1}{2}\left|\nabla u_{\tau}\right|^{2},
\end{aligned}
$$

where $\xi_{\tau} \in \partial I_{[0,1]}\left(\chi_{\tau}\right)$. Then, we prescribe

$$
f_{\tau} \rightarrow 0 \quad \text { in } L^{2}\left(0, T ; V^{\prime}\right)
$$

as $\tau \searrow 0$. We aim to investigate the asymptotics behavior of the solutions $\left(u_{\tau}, \chi_{\tau}\right)$, as the parameter $\tau \searrow 0$. The following theorem can be proved.

Theorem 4.1. Let (4.3) hold. Then, as $\tau \searrow 0$ the following convergences are verified

$$
\begin{aligned}
& u_{\tau} \stackrel{*}{\rightarrow} 0 \quad \text { in } H^{1}(0, T ; V) \cap L^{\infty}(0, T ; W), \\
& \left|\nabla u_{\tau}\right|^{2} \stackrel{*}{\rightarrow} d \geq 0 \quad \text { in } L^{\infty}(0, T ; H), \\
& \chi_{\tau} \stackrel{*}{\rightarrow} \chi \quad \text { in } H^{1}(0, T ; H) \cap L^{\infty}(0, T ; V) \cap L^{2}\left(0, T ; H^{2}(\Omega)\right) .
\end{aligned}
$$

Moreover, $d$ and $\chi$ fulfill, a.e. in $(0, T)$,

$$
\chi_{t}+A \chi+\xi=w-\frac{1}{2} d \text { in } H,
$$

for some $\xi$ belonging to $\partial I_{[0,1]}(\chi)$ a.e. in $Q$.

Proof. The proof of Theorem 4.1 is performed by exploiting an a priori estimates-passage to the limit procedure. In particular, we prove some estimates on the solutions which are uniform with respect to $\tau$, and then pass to the limit by compactness arguments. 
First a priori estimate. We test (4.2) by $\chi_{\tau_{t}}$ and (4.1) by $u_{\tau_{t}}$, integrate in time over $(0, t)$, and add the resulting equations. It is now a standard matter to integrate by parts and write

$$
\begin{aligned}
& \left\|u_{\tau_{t}}\right\|_{L^{2}(0, t ; V)}^{2}+\frac{1}{4} \sum_{i=1}^{3}\left\|D_{i} u_{\tau}(t)\right\|_{L^{4}(\Omega)}^{4}+\int_{0}^{t} \int_{\Omega} \chi_{\tau} \nabla u_{\tau} \cdot \nabla u_{\tau_{t}} \\
& +\left\|\chi_{\tau_{t}}\right\|_{L^{2}(0, t ; H)}^{2}+\frac{1}{2}\left\|\nabla \chi_{\tau}(t)\right\|_{H}^{2} \leq \int_{0}^{t}\left\|f_{\tau}\right\|_{V^{\prime}}\left\|u_{\tau_{t}}\right\|_{V} d s \\
& +\int_{0}^{t} \int_{\Omega} w \chi_{\tau_{t}}-\frac{1}{2} \int_{0}^{t} \int_{\Omega}\left|\nabla u_{\tau}\right|^{2} \chi_{\tau_{t}} .
\end{aligned}
$$

Notice that on the left hand side we can equivalently rewrite (cf. (3.4))

$$
\begin{aligned}
& \int_{0}^{t} \int_{\Omega} \chi_{\tau} \nabla u_{\tau} \cdot \nabla u_{\tau_{t}}=\frac{1}{2} \int_{0}^{t} \int_{\Omega} \chi_{\tau} \frac{d}{d t}\left|\nabla u_{\tau}\right|^{2} \\
& \quad=\frac{1}{2} \int_{\Omega} \chi_{\tau}(t)\left|\nabla u_{\tau}(t)\right|^{2}-\frac{1}{2} \int_{0}^{t} \int_{\Omega} \chi_{\tau_{t}}\left|\nabla u_{\tau}\right|^{2},
\end{aligned}
$$

so that two terms cancel and applying the Young inequality, we finally obtain

$$
\begin{aligned}
& \left\|u_{\tau_{t}}\right\|_{L^{2}(0, t ; V)}^{2}+\sum_{i=1}^{3}\left\|D_{i} u_{\tau}(t)\right\|_{L^{4}(\Omega)}^{4}+\int_{\Omega} \chi_{\tau}(t)\left|\nabla u_{\tau}(t)\right|^{2} \\
& +\left\|\chi_{\tau_{t}}\right\|_{L^{2}(0, t ; H)}^{2}+\left\|\nabla \chi_{\tau}(t)\right\|_{H}^{2} \leq c\left(1+\left\|f_{\tau}\right\|_{L^{2}\left(0, T ; V^{\prime}\right)}^{2}\right) .
\end{aligned}
$$

Remark 4.2. In particular, notice that the presence of the higher order elasticity term allows us to bound $\left|\nabla u_{\tau}\right|^{2}$ in $L^{2}(Q)$ in (4.2) after prescribing only that $f_{\tau}$ is bounded in $L^{2}\left(0, T ; V^{\prime}\right)$ (cf. Remark 3.2).

Second a priori estimate. We proceed formally and test (4.2) by $A \chi_{\tau}$. After integrating over $(0, t)$, by monotonicity of the subdifferential operator, standard arguments show that (cf. (4.10))

$$
\left\|\nabla \chi_{\tau}(t)\right\|_{H}^{2}+\left\|A \chi_{\tau}\right\|_{L^{2}(0, t ; H)}^{2} \leq c\left(1+\left\|\nabla u_{\tau}\right\|_{L^{4}(Q)}^{4}\right) \leq c .
$$

Finally, a comparison in (4.2) yields

$$
\left\|\xi_{\tau}\right\|_{L^{2}(0, T ; H)} \leq c .
$$


For the sake of simplicity, we summarize the main estimates we have obtained and we exploit in the following passage to the limit argumentation,

$$
\begin{aligned}
& \left\|u_{\tau}\right\|_{H^{1}(0, T ; V) \cap L^{\infty}(0, T ; W)} \leq c, \\
& \left\|\left|\nabla u_{\tau}\right|^{2}\right\|_{L^{\infty}(0, T ; H)} \leq c, \\
& \left\|\chi_{\tau}\right\|_{H^{1}(0, T ; H) \cap L^{\infty}(0, T ; V) \cap L^{2}\left(0, T ; H^{2}(\Omega)\right)} \leq c .
\end{aligned}
$$

Thus, well known weak and weak star compactness results allow us to deduce that the following convergences hold, at least up to the extraction of a suitable subsequence

$$
\begin{aligned}
& u_{\tau} \stackrel{*}{\rightarrow} u \quad \text { in } H^{1}(0, T ; V) \cap L^{\infty}(0, T ; W), \\
& \left|\nabla u_{\tau}\right|^{2} \stackrel{*}{\rightarrow} d \geq 0 \quad \text { in } L^{\infty}(0, T ; H), \\
& \chi_{\tau} \stackrel{*}{\rightarrow} \chi \quad \text { in } H^{1}(0, T ; H) \cap L^{\infty}(0, T ; V) \cap L^{2}\left(0, T ; H^{2}(\Omega)\right) .
\end{aligned}
$$

In particular, let us observe that the weak convergence (4.16) is not sufficient to identify $d$ with $|\nabla u|^{2}$ in (4.17). Hence, by (4.12) we also infer that

$$
\xi_{\tau} \rightarrow \xi \quad \text { in } L^{2}(0, T ; H)
$$

Moreover, by compactness, from (4.16) and (4.18), we obtain the following strong convergences (actually something more holds)

$$
\begin{aligned}
& u_{\tau} \rightarrow u \quad \text { in } C^{0}([0, T] ; H), \\
& \chi_{\tau} \rightarrow \chi \quad \text { in } C^{0}([0, T] ; H) \cap L^{2}(0, T ; V) .
\end{aligned}
$$

Owing to the results presented in [2], (4.19) and (4.21) enable us to identify at the limit $\xi \in \partial I_{[0,1]}(\chi)$. Thus, (4.16)-(4.18), (4.19), and (4.20)-(4.21), allow us to pass to the limit as $\tau \searrow 0$ in (4.1) and (4.2) and get, a.e. in $(0, T)$,

$$
\begin{aligned}
& u_{t}+A u_{t}+\mathcal{A} u+B(\chi \nabla u)=0 \quad \text { in } V^{\prime}, \\
& \chi_{t}+A \chi+\xi=w-\frac{1}{2} d \quad \text { in } H
\end{aligned}
$$

where

$$
\xi \in \partial I_{[0,1]}(\chi) \quad \text { a.e. in } Q .
$$


Then, as one can prove that (4.22)-(4.23) admits a unique solution (cf. the proof of Theorem 3.1), we can identify $u=0$, so that (4.22) vanishes, namely there are not macroscopic displacements or deformations at the limit. Hence, let us discuss the mechanical meaning of $d$ in (4.23). By (4.17) it follows that $d$ is the weak limit of deformation energies associated to the vanishing sequence of macroscopic motions (cf. (4.16)) and, in general, $d \neq 0$. Thus, this function, representing a source of damage in (4.23), can be interpreted as the remaining damaging effect of macroscopic motions, acting at a microscopic level. It follows that a sequence of vanishing macroscopic motions can retain its damaging effect, at the limit, as a source of damage in the equation of microscopic motions. In order to support this fact, in the next section we briefly discuss the balance of the energy of our problem.

\section{Balance of the energy}

We first consider the work provided by the exterior forces during a time interval $(0, t)$, which is given by

$$
T_{\tau}(t)=\int_{0}^{t}\left\langle f_{\tau}, u_{\tau_{t}}\right\rangle
$$

By (4.1), we can rewrite (5.1) as it follows

$$
\begin{aligned}
T_{\tau}(t)= & \int_{0}^{t} \int_{\Omega}\left|u_{\tau_{t}}\right|^{2}+\int_{0}^{t} \int_{\Omega}\left|\nabla u_{\tau_{t}}\right|^{2} \\
& +\sum_{i=1}^{3} \frac{1}{4} \int_{\Omega}\left|D_{i} u_{\tau}(t)\right|^{4}+\frac{1}{2} \int_{0}^{t} \int_{\Omega} \chi_{\tau} \frac{d}{d t}\left|\nabla u_{\tau}\right|^{2} .
\end{aligned}
$$

We let

$$
A_{\tau}(t)=\int_{0}^{t} \int_{\Omega}\left|u_{\tau_{t}}\right|^{2}+\int_{0}^{t} \int_{\Omega}\left|\nabla u_{\tau_{t}}\right|^{2}+\sum_{i=1}^{3} \frac{1}{4} \int_{\Omega}\left|D_{i} u_{\tau}(t)\right|^{4},
$$


and then, by (4.2), we write

$$
\begin{aligned}
& \frac{1}{2} \int_{0}^{t} \int_{\Omega} \chi_{\tau} \frac{d}{d t}\left|\nabla \chi_{\tau}\right|^{2}=\frac{1}{2} \int_{\Omega} \chi_{\tau}(t)\left|\nabla u_{\tau}(t)\right|^{2} \\
& \quad-\frac{1}{2} \int_{0}^{t} \int_{\Omega} \chi_{\tau_{t}}\left|\nabla u_{\tau}\right|^{2}=\frac{1}{2} \int_{\Omega} \chi_{\tau}(t)\left|\nabla u_{\tau}(t)\right|^{2}+\int_{0}^{t} \int_{\Omega}\left|\chi_{\tau_{t}}\right|^{2} \\
& \quad+\frac{1}{2} \int_{\Omega}\left|\nabla \chi_{\tau}(t)\right|^{2}-\int_{0}^{t} \int_{\Omega} w \chi_{\tau_{t}} .
\end{aligned}
$$

In particular, in (5.4) we have used the fact that

$$
\int_{0}^{t} \int_{\Omega} \xi_{\tau} \chi_{\tau_{t}}=\int_{\Omega} I_{[0,1]}\left(\chi_{\tau}(t)\right)-\int_{\Omega} I_{[0,1]}\left(\chi_{\tau}(0)\right)=0,
$$

as $\chi_{\tau} \in[0,1]$ a.e. and $\chi_{\tau}(0)=1$. Hence, we set

$$
B_{\tau}(t)=\int_{0}^{t} \int_{\Omega}\left|\chi_{\tau_{t}}\right|^{2}+\frac{1}{2} \int_{\Omega}\left|\nabla \chi_{\tau}(t)\right|^{2}-\int_{0}^{t} \int_{\Omega} w \chi_{\tau_{t}} .
$$

Thus, we can rewrite $T_{\tau}$ as follows

$$
T_{\tau}(t)=A_{\tau}(t)+B_{\tau}(t)+\frac{1}{2} \int_{\Omega} \chi_{\tau}(t)\left|\nabla u_{\tau}(t)\right|^{2} .
$$

Then, we investigate the limit as $\tau \searrow 0$ of $T_{\tau}$. By (4.18), the weak lower semicontinuity of norms, and (4.23) we can infer that

$$
\begin{aligned}
\liminf _{\tau \searrow 0} B_{\tau}(t) & =Q_{B}(t)+\int_{0}^{t} \int_{\Omega}\left|\chi_{t}\right|^{2}+\frac{1}{2} \int_{\Omega}|\nabla \chi(t)|^{2}-\int_{0}^{t} \int_{\Omega} w \chi_{t} \\
& =Q_{B}(t)-\frac{1}{2} \int_{0}^{t} \int_{\Omega} d \chi_{t}=Q_{B}(t)+\mathcal{D}(t),
\end{aligned}
$$

where $Q_{B} \geq 0$. Analogously, by (4.16)-(4.17) (where $u=0$ ) we have that

$$
\liminf _{\tau \searrow 0} A_{\tau}(t)=Q_{A}(t)+\frac{1}{4}\|d(t)\|_{H}^{2},
$$

and $Q_{A} \geq 0$. Finally, by (4.17) and (4.21) we have

$$
\lim _{\tau \searrow 0} \frac{1}{2} \int_{\Omega} \chi_{\tau}(t)\left|\nabla u_{\tau}(t)\right|^{2}=\frac{1}{2} \int_{\Omega} \chi(t) d(t) .
$$


Hence, we can take a suitable subsequence of $\tau_{j}$ such that

$$
\lim _{\tau_{j} \searrow 0} T_{\tau_{j}}(t)=\liminf _{\tau \searrow 0} T_{\tau}(t)
$$

Thus, denoting by

$$
T(t)=\lim _{\tau_{j} \searrow 0} T_{\tau_{j}}(t),
$$

owing to (5.8)-(5.9) we can eventually infer that

$$
T(t)=Q(t)+\mathcal{D}(t)+S(t)
$$

where (cf. (5.8) and (5.9))

$$
Q(t)=Q_{A}(t)+Q_{B}(t)
$$

$\mathcal{D}$ is introduced in (5.8), and

$$
S(t)=\frac{1}{4}\|d(t)\|_{H}^{2}+\frac{1}{2} \int_{\Omega} \chi(t) d(t) .
$$

Thus, let us conclude that the work which is provided to the structure $T(t)$ is divideed between damaging external work $\mathcal{D}(t)$, external source of heat $Q(t)$, and stored energy $S(t)$. In particular, when no instantaneous damage work is applied at the final time $t(d(t)=0)$, from (5.15) it results $S(t)=0$ and, consequently, the work which has been provided is exactly the sum of the damaging work $\mathcal{D}$ and of the heat sources $Q$ resulting from the dissipative phenomena.

\section{REFERENCES}

[1] C. Baiocchi, Sulle equazioni differenziali astratte lineari del primo e del secondo ordine negli spazi di Hilbert, Ann. Mat. Pura Appl. (IV) 76 (1967), 233-304.

[2] V. Barbu, Nonlinear Semigroups and Differential Equations in Banach Spaces, Noordhoff, Leyden, (1976).

[3] E. Bonetti and G. Schimperna, Local Existence to Fremond's Model for Damaging in Elastic Materials, preprint IMATI-CNR, Pavia (Italy) n. (2002), to appear in Continuum Mechanics and Thermodynamics.

[4] M. Frémond, Non-smooth Thermomechanics, Springer-Verlag, Berlin, (2001).

[5] M. Frémond, Damage theory. A macroscopic effect vanishes but its effects remain, Comp. Appl. Math. 21 (2002), 1-14. 
[6] M. Frémond, K.L. Kuttler, B. Nedjar and M. Shillor, One dimensional models of damage, Adv. Math. Sci. Appl. 8 (1998), 541-570.

[7] M. Frémond, K.L. Kuttler and M. Shillor, Existence and uniqueness of solutions for a dynamic one-dimensional damage model, J. Math. Anal. Appl. 229 (1999), 271-294.

[8] M. Frémond and B. Nedjar, Damage, gradient of damage, and principle of virtual power, Internat. J. Solids Structures 33 (1996), 1083-1103.

[9] J.L. Lions, Quelques Méthodes de Résolution des Problèmes aux Limites non Linéaires, Dunod, Gauthier-Villars Paris, (1969).

[10] J.L. Lions and E. Magenes, Non-homogeneous Boundary alue Problems and Applications, Vol. I, Springer-Verlag, Berlin, (1972).

[11] J.J. Moreau, Sur les lois de frottement, de viscosité et de plasticité, C.R. Acad. Sci. 27 (1970), 608-611.

[12] B. Nedjar, Mécanique de l'endommagement. Théorie du premier gradient et application au béton, PhD. thesis, Ecole Nationale des Ponts et Chaussées, Paris, (1995).

[13] J. Simon, Compact sets in the space $L^{p}(0, T ; B)$, Ann. Mat. Pura Appl. (4) 146 (1987), 65-96. 